Special issue of the 2nd International Conference on Computational and Experimental Science and Engineering (ICCESEN 2015)

\title{
Industrial PC Based Heliostat Control for Solar Power Towers
}

\author{
O.M. PIŞIRIR ${ }^{a, *}$ AND O. BINGÖL ${ }^{b}$ \\ ${ }^{a}$ Süleyman Demirel University, Institute of Natural and Applied Sciences, Isparta, Turkey \\ ${ }^{b}$ Süleyman Demirel University, Faculty of Technology Electrical and Electronics Engineering, Isparta, Turkey
}

Nowadays, fossil fuels are being used widely and are being rapidly depleted. Therefore, alternative energy sources are needed. In solar energy systems, renewable energy projects have gained importance and have been produced. As a system that is being developed in recent years, solar power towers have been built in many countries and more and more people make use of them. Heliostats are some of the important components of the system which provide energy by focusing the sun rays. In this study, we have investigated the control of heliostats which are used in solar power towers. Industrial computers are mainly used in heliostats. The embedded operating systems, which are built into them, are flexible and can operate improved software and databases. By connecting a database and electronic control circuits to an industrial computer we were able to build a prototype heliostat. Given two angle values which specify the target's direction we were able to track the movements of the sun, have the heliostat work properly and analyse the results.

DOI: 10.12693/APhysPolA.130.36

PACS/topics: 88.40.fp

\section{Introduction}

Around the world the energy systems are mainly based on fossil fuels. However, in the near future, as a result of excessive use, the sources of fuel will start to decrease and this will lead to a search of new reserves and invention of new technologies. In recent years, energy resources have great importance for sustainable production. According to many people all around the world, solar energy is economic, nature friendly, and sustainable. Therefore, many countries are interested in solar energy. Our country, as a result of its geographical location, has a great potential to produce and use solar energy. It has yearly about 2623 hours of sunshine. $63 \%$ of its area receives sunshine for 10 months of an year and $17 \%$ can use the sunlight during the entire year. Every year the amount of energy produced from sunlight is increasing [1-3].

Solar power towers are systems that transform solar energy to electric energy. In this system thousands of mirrors are focused to a tower. The system of mirrors is called "heliostat". Heliostats, with the aid of computer control focus the solar rays directly to a spot on the top of the tower. They can move vertically and horizontally.

When they start to function, first they work in search mode and find the target, once they lock on the sunlight they switch to follow mode. Thus they can transmit the sunlight to the receiver which is located on the top of the tower. Heliostats usually work in the open loop mode and the required angles for their positioning are found through astronomical calculations. Therefore, it is very important to follow the focus point in these systems. At the focus point a receptor is installed. The temperature at this point can reach up to $1000^{\circ} \mathrm{C}$. Hot air heated

\footnotetext{
* corresponding author; e-mail: yl1130130015@stud.sdu.edu.tr
}

by molten salt moves to the turbine where the electricity is produced [4-6]. A solar power tower is shown in Fig. 1.

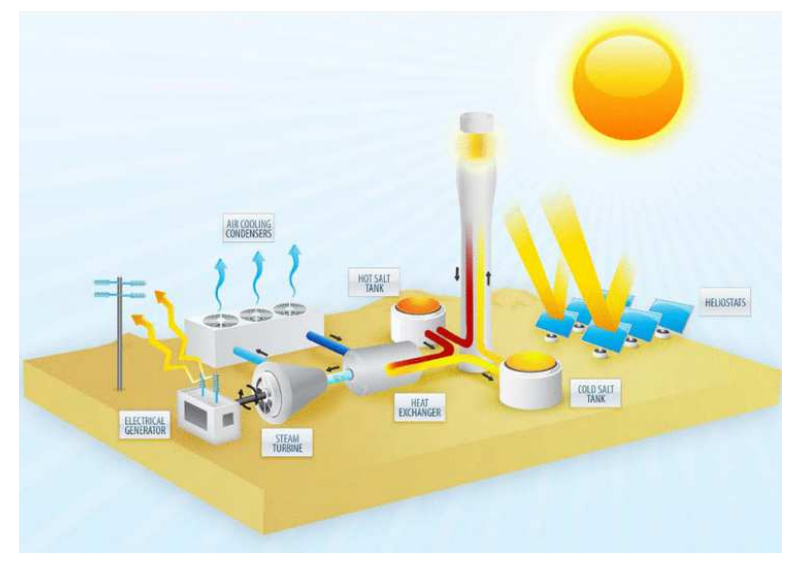

Fig. 1. Solar power tower [7].

In literature, eSolar company has been able to operate the heliostats structure and achieve positioning by hybrid step engines without sensors [8]. The closed loop control of the heliostats is substantially decreasing the aiming faults [9]. The daily, monthly and yearly positions of the Sun can be calculated by developing MATLAB based software and economical solutions can be developed by systems controlled by PLC [10]. The required calculations and models for directing light, coming from unlevelled surfaces and reaching the target in heliostats, have been studied [11]. The positioning of the heliostats has been analysed and required calculations have been performed so they don't overshadow each other [12]. The technical sun-tracking errors of heliostats have been also mathematically analysed [13]. Parameters required for setting up a solar power tower, like the positioning of the mirrors, area radius, the heights of the towers and the cost analysis, have been approached by another study [14]. 
In this study, a prototype heliostat for solar power towers is designed and produced. The control of the heliostat has been provided by a software and database working on an industrial computer. Industrial computer, Advantech ARK-1388V, was used in this study. It is able to run software which can be visually programmed through an embedded operating system and also can function as remote controlling system (3G, GPRS or WIFI). Through these specifications of the industrial computer, a practical solution both for troubleshooting and control of the heliostats is provided.

\section{Materials and methods}

\subsection{System design and implementation}

The general system structure for control of the heliostat consists of three parts, the mechanical design, the software design and the actuator circuit design. The mechanical design consists of such elements as encoder, step motor, redactor. The software design consists of such elements as database and HeliosControl software. The actuator circuit design consists of such elements as relays, PLC, clips. The block diagram of the system is shown in Fig. 2.

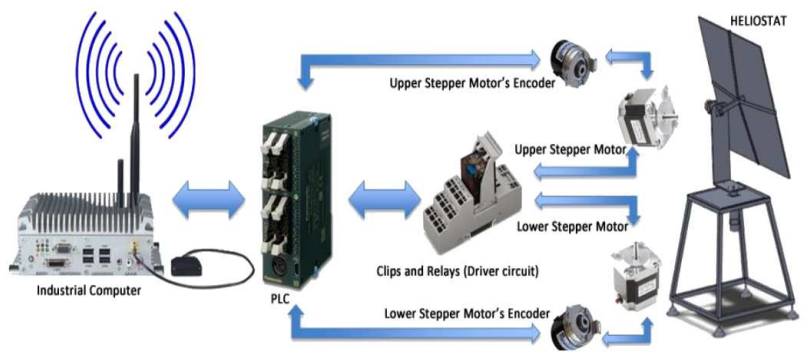

Fig. 2. System block diagram.

\subsection{Mechanical design}

The designed system can carry a $1 \times 1 \mathrm{~m}^{2}$ mirror. If demanded, control of heliostats with bigger dimension can be provided. The mechanical design which consists of mirror, stabilizer weight, reducer, stepper motor and encoder can be seen in Fig. 3. The reducer used in the system reduces the 1.8 degree step angle of the stepper motor by $1 / 5$ to 0.36 degrees and provides precise positioning. Figure 4 shows the entire heliostat system.

\subsection{Software design}

ARK 1388V industrial computer, produced by Advantech company, is a fanless computer, having embedded computing systems, which can perform with limited power requirements. It includes Core 2 Duo $1.06 \mathrm{GHz}$ processor. It can work between the temperatures of 20 and $60^{\circ} \mathrm{C}$ with an effective thermal design. It includes four serial interfaces, LVDS and VGA independent display interfaces, four USB ports, one LAN port, digital input and output interfaces, voice output and rich variety of inputs and outputs. It includes safe boot and
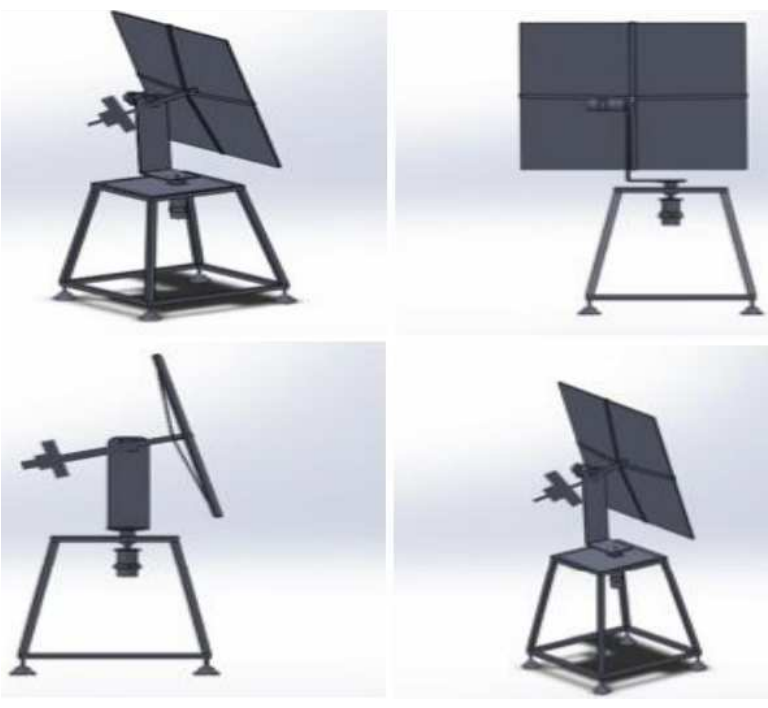

Fig. 3. Heliostat design.

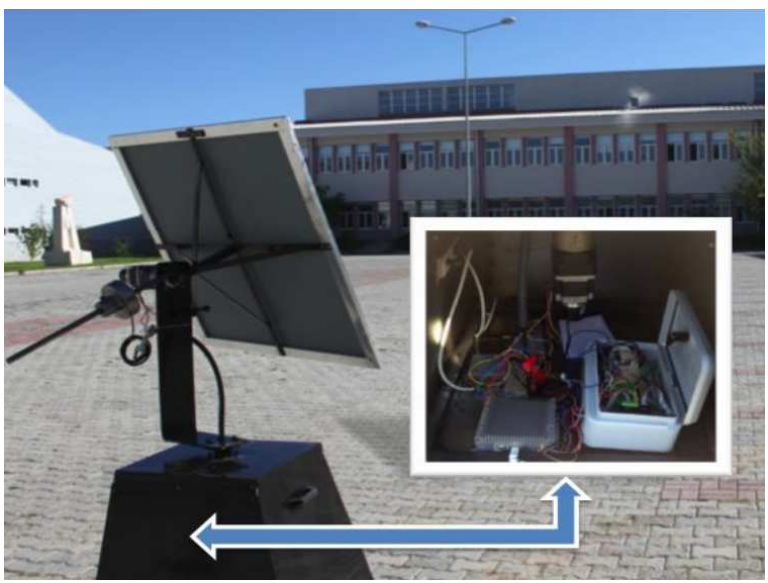

Fig. 4. Heliostat system.

shutdown functions. In case when system is not responding, it can restart the system without any intervention, using the watchdog timer feature. Therefore it is suitable to be used in many fields. It can perform with $12 \mathrm{~V}$ and $24 \mathrm{~V}$. It also includes the characteristics of the protection of hardware power, smart software display and checking. Passive cooling system provides silent operation and prevents defects arising from breakdown of the cooling. 802.11b/g WLAN card, GPS receiver which supports 50 channels, GPRS support, 3.5G antenna module, provide the growing wireless network functions and allows $3 \mathrm{G}$ connection. The industrial computer is shown in Fig. 5. The reasons of using industrial computer in our study are to provide consistency, to perform outdoors, at high temperatures due to passive cooling system, to have a compact built, portability, possibility of remote control, to provide modularity of the systems, fast operation, visual programming options with embedded computing system. Industrial computers, with these characteristics, provide a practical solution in terms of remote observation and control of heliostats. 


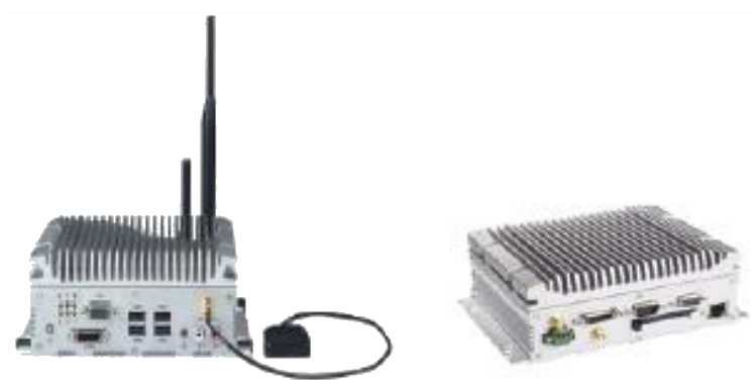

Fig. 5. Industrial computer.

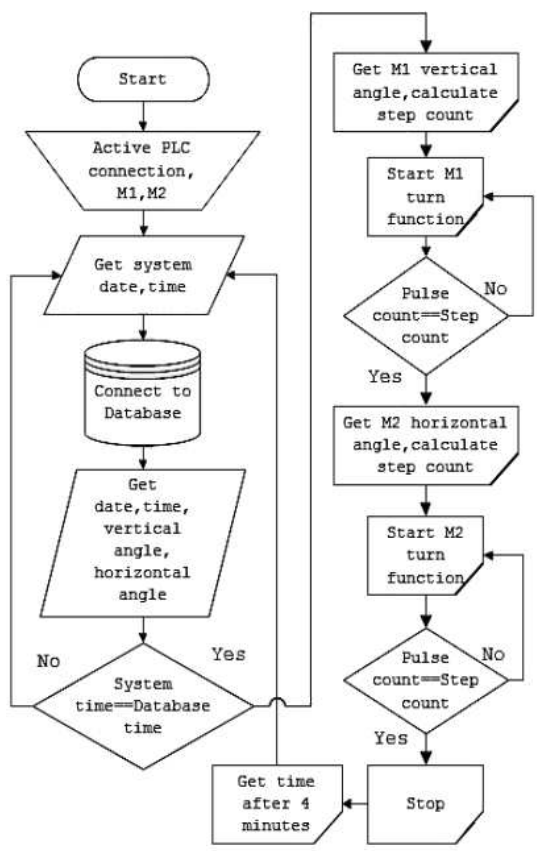

Fig. 6. HeliosControl software algorithm.

The software connects to the database every four minutes, and receives the vertical and horizontal positions for the heliostat at that time and it also receives the date and time. After that it sends the required commands to run the step motors to the PLC and the software on the PLC checks the driver circuit and provides the movement of the motors. HeliosControl algorithm is shown in Fig. 6.

\subsection{Motor driver circuit design}

The actuator circuit design consists of relays, PLC, clips and a connection box. Relays are used to control the stepper motor coils and eight solid state relays (SSR). The reason of using SSRs is faster operation and opening/closing without an arc, compared to conventional relays. The $24 \mathrm{~V}$ power supply is used for PLC, connection box and encoders. $12 \mathrm{~V}$ power supply is used for stepper motors. Each relay is connected to the outputs of the PLC by clips. These control the step coils one by one over the contacts through signals coming from the PLC outputs. Figure 7 shows the motor drive circuit and Table I shows the elements.

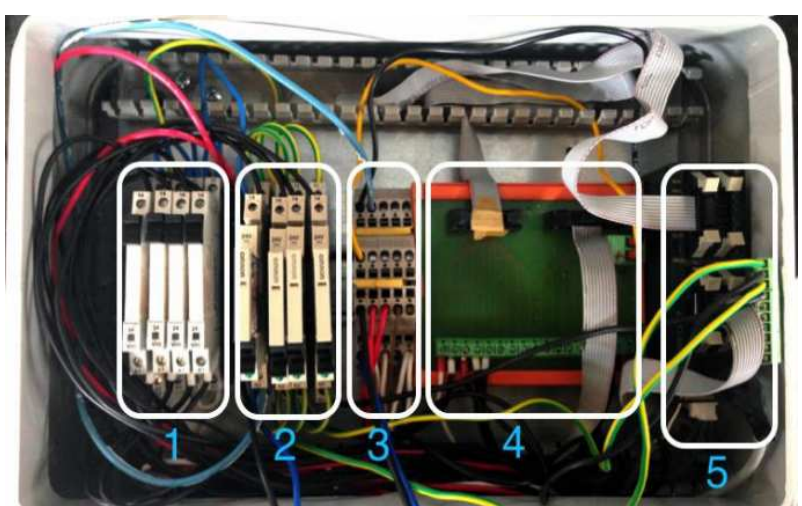

Fig. 7. Motor driver circuit.

TABLE I

The elements of the circuit.

\begin{tabular}{c|c}
\hline \hline 1 & Relays of the vertical movement motor \\
2 & Relays of the horizontal movement motor \\
3 & +12 and +24 volt clips \\
4 & Connection box \\
5 & PLC (Panasonic FP0R-C32CP)
\end{tabular}

In this study, Panasonic FP0R-C32CP model PLC is used. The most important advantages of using the PLC are its high-speed counter and pulse output features and its Modbus support. Modbus is transmission and communication protocol which we use to control stepper motors. It is an ideal PLC considering its speed and flexibility features. Panasonic FPWIN Pro software was used for programming the PLC. PLC controlled motor drive circuit is shown in Figs. 8 and 9. Figure 10 also shows software that is running on the PLC.

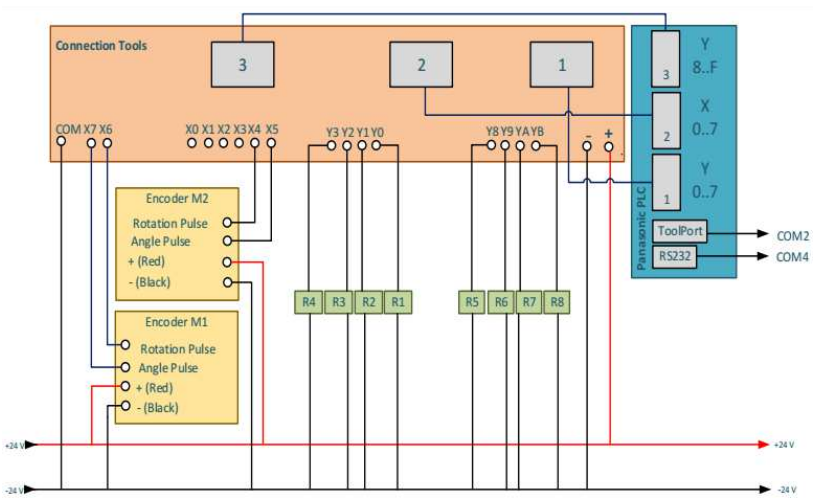

Fig. 8. Motor driver circuit diagram.

The "step number" data coming from the HeliosControl software goes to the PLC and the PLC software starts to run. The running of the step motors continues until the "step number" and the "pulse count" coming from the encoder are equalizes. The heliostat keeps it position for four minutes. After four minutes, the software receives the new angle values and if there is a change, applies the 


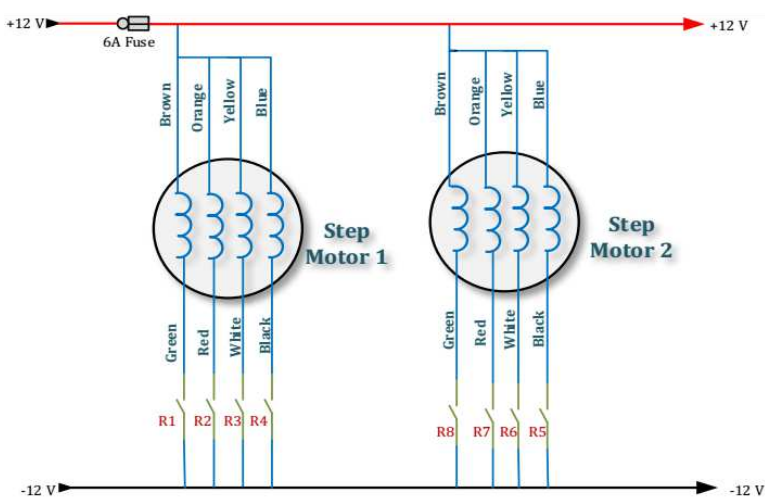

Fig. 9. Driver circuit motor diagram.

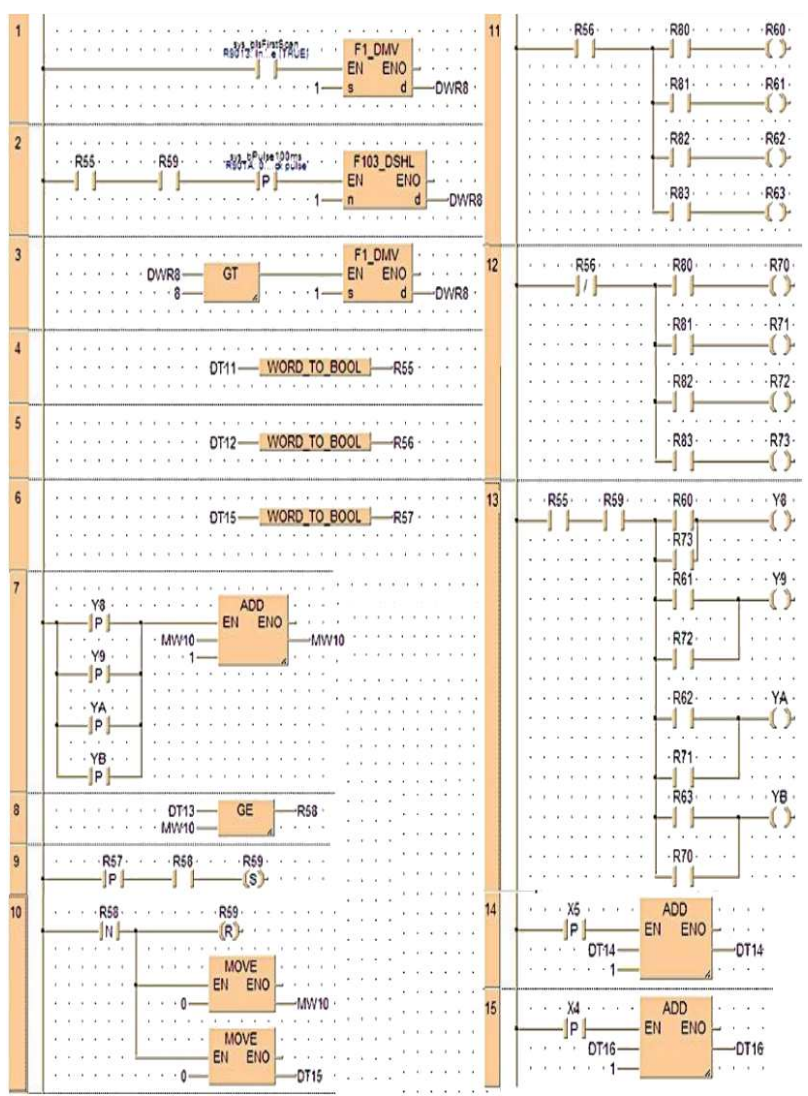

Fig. 10. PLC software.

same procedures. It calculates the step number using new angle value and the previous angle value and the procedure of redirecting the motors starts again.

In Fig. 11a, the angles, received from the database by HeliosControl at 14:48, are shown. When the angles change at 14:52, firstly the vertical angle is located as seen in Fig. 11b. After the motor which makes vertical positioning stops, the motor making the horizontal positioning starts to operate within the same minute, and it completes its location process as can be seen in Fig. 11c. While the motors perform the positioning, their current positions are shown in Figs. 11b and 11c differently.

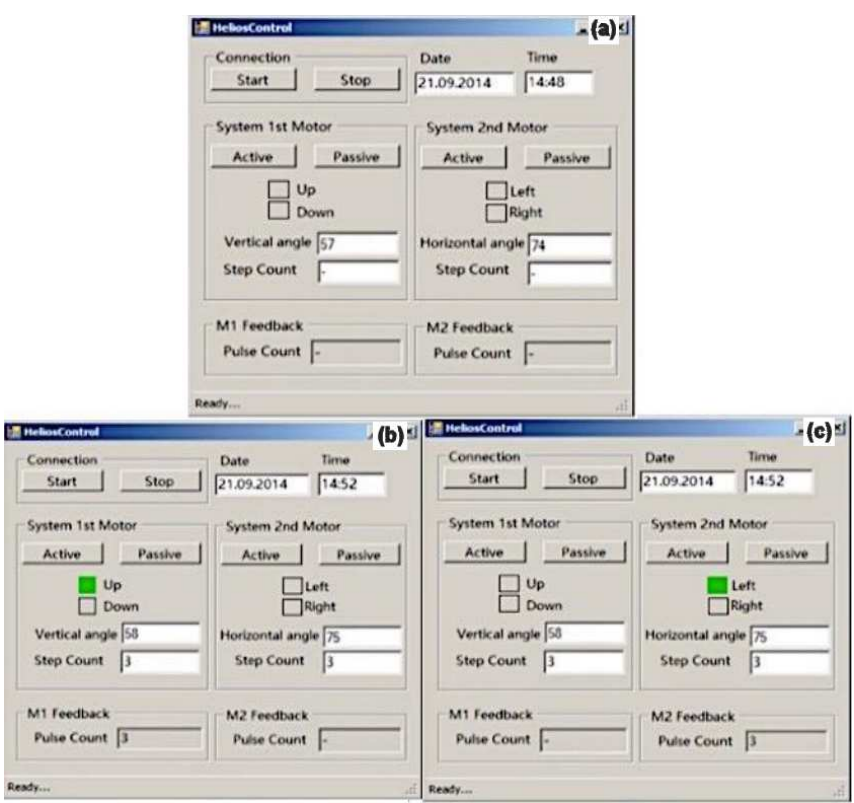

Fig. 11. Timing and motor control by HeliosControl program.

\section{Conclusions}

To test the system, building E-12, located in the Isparta Suleyman Demirel University West Campus, has been used as a simulated tower. The heliostat was tested during different periods of the year. The location info of the test area and the heliostat has been previously calculated and the angle values at these points were transferred to the database. During the tests that were realized between 09:00 and 18:00, all elements that control the heliostat were evaluated. Although the industrial computer worked outdoor, it continued to work stable with its temperature reaching $60^{\circ} \mathrm{C}$. The embedded operating system of the industrial computer allowed us to remotely connect and control all operations in real time. When there was a need for updating of angle values in the database, the system had quickly adapted to new values, without needing reprogramming, which gives a great advantage. Figure 12 shows the imaginary tower

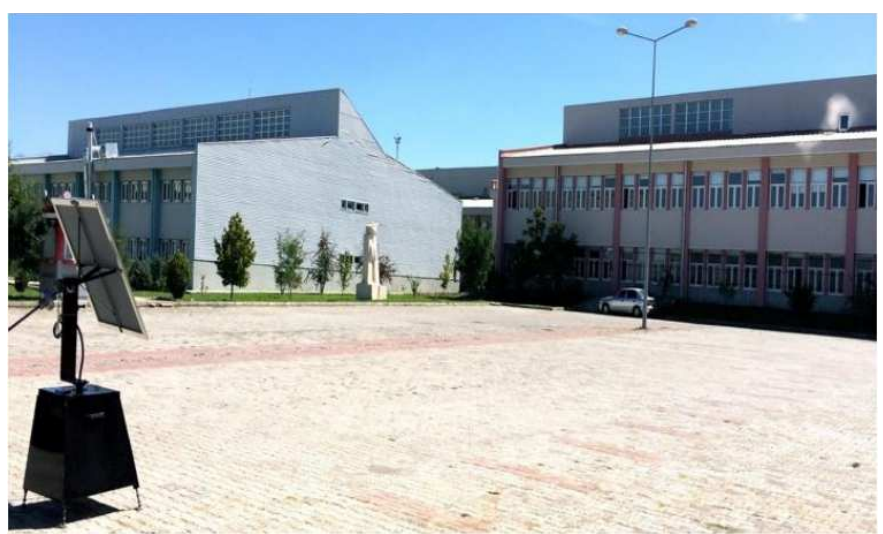

Fig. 12. Heliostat and the imaginary tower. 


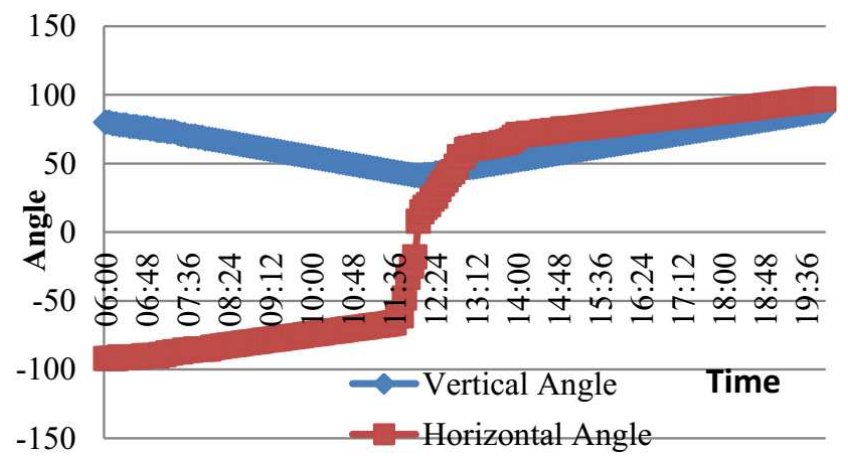

Fig. 13. Angle values during a day.

and heliostat at the experimental area. Figure 13 shows the angle information received by the HeliosControl from the database during one day.

Our study will also draw attention in our country to renewable energy projects that may replace the fossil fuels, because our country benefits from the sunshine most of the year. Such projects are very important and valuable to meet the increasing demand for energy. Such facilities will have environmental and economic benefits for the country.

\section{Acknowledgments}

This work was funded by a grant (No. 3451-YL1-13) from the Scientific Research Projects of Suleyman Demirel University.

\section{References}

[1] M. Cetin, N. Egrican, Energy Policy 39, 7184 (2011).

[2] M. Ocak, Z. Ocak, S. Bilgen, S. Keles, K. Kaygusuz, Energ. Convers. Manage. 45, 845 (2004).
[3] G. Boyle, B. Everet, J. Ramage, Energy Systems and Sustainability, Oxford University Press, Oxford 2003.

[4] DGS (German section of the International Solar Energy Society), Planning and Installing Solar Thermal Systems: A Guide for Installers, Architects and Engineers, Routledge, New York 2010.

[5] H.P. Garg, J. Prakash, Solar Energy: Fundamentals and Applications, 1st ed., McGraw Hill Education, New Delhi 2000.

[6] F. Xu, W. Liang, in: 11th Int. Conf. Control, Automation, Robotics and Vision, Singapore 2010, p. 2284.

[7] Univ. of Pittsburg, 2016, [Online]. Available: www . pitt.edu/ viv14/images/6035949-fig-8-large. gif.

[8] S. Schell, Sol. Energ. 85, 614 (2011).

[9] A. Kribus, I. Vishnevetsky, A. Yogev, T. Rubinov, Energy 29, 905 (2004).

[10] A. Chaib, M. Kesraoui, E. Kechadi, in: 8th Int. Conf. and Exhibition on Ecological Vehicles and Renewable Energies, Monte Carlo, 2013, p. 1.

[11] X. Wei, Z. Lu, W. Yu, H. Zhang, Z. Wang, Renew. Energ. 36, 2687 (2011).

[12] F.J. Collado, J. Guallar, Renew. Sust. Energ. Rev. 20, 142 (2013).

[13] V. Badescu, Sol. Energ. 82, 1192 (2008).

[14] R. Senol, I. Ucgul, A. Koyun, M. Acar, J. Faculty Eng. Architect. Gazi Univ. 26, 813 (2011). 